\title{
Importance of Anatomical Efficacy for Disease Control in Neovascular AMD: An Expert Opinion
}

\author{
Konstantinos Balaskas · Winfried M. Amoaku · Tomas Cudrnak • \\ Louise M. Downey • Markus Groppe · Sajjad Mahmood • \\ Hemal Mehta • Quresh Mohamed · Bushra Mushtaq · Philip Severn • \\ Athanasios Vardarinos • Yit C. Yang
}

Received: February 25, 2021 / Accepted: March 24, 2021 / Published online: April 10, 2021

(C) The Author(s) 2021

\section{ABSTRACT}

Background: Neovascular age-related macular degeneration (nAMD) presents a significant treatment burden for patients, carers and medical retina services. However, significant debate remains regarding how best to manage nAMD when assessing disease activity by optical

\section{K. Balaskas $(\bowtie)$}

NIHR Biomedical Research Centre at Moorfields Eye Hospital NHS Foundation Trust and University College of London Institute of Ophthalmology, London, UK

e-mail: k.balaskas@nhs.net

W. M. Amoaku

Academic Ophthalmology, Division of Clinical

Neuroscience, University of Nottingham,

Nottingham, UK

T. Cudrnak

Royal Eye Infirmary, University Hospitals Plymouth NHS Trust, Plymouth, UK

\section{M. Downey}

Hull University Teaching Hospital, Hull, UK

\section{Groppe}

Ophthalmology, Stoke Mandeville, Buckinghamshire Healthcare NHS Trust, Aylesbury, UK

S. Mahmood

Manchester Royal Eye Hospital, Manchester

University NHS Foundation Trust, University of

Manchester, Manchester Academic Health Science

Centre, Manchester, UK coherence tomography (OCT), and particularly the significance of different types of fluid and how the understanding of anatomical efficacy can influence treatment strategies. This article provides opinion on the practical implications of anatomical efficacy and significance of fluid in the management of nAMD and proposes recommendations for healthcare professionals

\section{H. Mehta}

Royal Free London NHS Foundation Trust, London, UK

Q. Mohamed

Gloucestershire Eye Unit, Cheltenham General Hospital, Gloucestershire Hospitals NHS Foundation Trust, Gloucester, UK

\section{B. Mushtaq}

Birmingham and Midland Eye Centre, Sandwell and West Birmingham NHS Trust, Birmingham, UK

\section{P. Severn}

South Tees Hospitals NHS Foundation Trust, Middlesbrough, UK

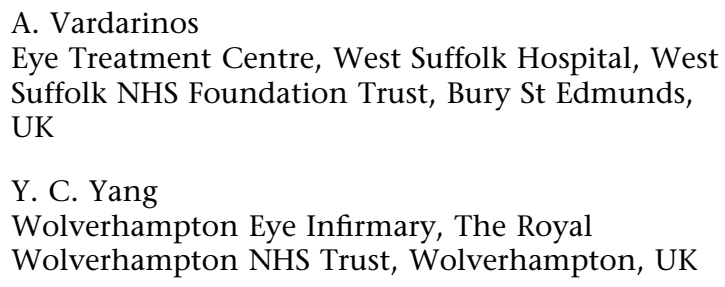

A. Vardarinos

Eye Treatment Centre, West Suffolk Hospital, West Suffolk NHS Foundation Trust, Bury St Edmunds, UK

Y. C. Yang

Wolverhampton Eye Infirmary, The Royal Wolverhampton NHS Trust, Wolverhampton, UK 
(HCPs) to improve understanding and promote best practice to achieve disease control.

Methods: An evidence-based review was performed and an expert panel debate from the Retina Outcomes Group (ROG), a forum of retinal specialists, provided insights and recommendations on the definition, role and practical implications of anatomical efficacy and the significance of fluid at the macula in the management of nAMD.

Results: The ROG has developed recommendations for achieving disease control through a zero-tolerance approach to the presence of fluid in nAMD as patients who avoid fluctuations in fluid at the macula have better visual outcomes. Recommendations cover five key areas: service protocol, training, regimen, multidisciplinary teams and engagement. This approach facilitates more standardised protocol-based treatment strategies.

Conclusions: Targeting a fluid-free macula and aiming for disease control are essential to improve outcomes. As new therapies and technologies become available, drying the macula and maintaining disease control will become even more achievable. The outlined recommendations aim to promote best practice among HCPs and medical retina services to improve patient outcomes.

Keywords: Anatomical efficacy; Disease control fluid; OCT; Stability

\section{Key Summary Points}

Neovascular age-related macular degeneration (nAMD) presents a significant treatment burden for patients, carers and medical retina services.

However, significant debate remains regarding how best to manage nAMD when assessing disease activity, the significance of different types of fluid and how the understanding of anatomical efficacy can influence treatment strategies.

\section{DIGITAL FEATURES}

This article is published with digital features, including a summary slide, to facilitate understanding of the article. To view digital features for this article go to https://doi.org/10.6084/ m9.figshare.14290556.

\section{INTRODUCTION}

Age-related macular degeneration (AMD) is the leading cause of certified vision loss in the UK. The estimated number of new cases of neovascular AMD (nAMD) is around 40,000 each year, and the prevalence of nAMD is increasing [1-4]. If untreated, nAMD can cause rapid deterioration of vision via choroidal neovascularisation (CNV) $[1,5,6]$. Vascular endothelial growth factor (VEGF) has been linked to the development of the new blood vessels that are seen in CNV [1]. The risk of developing late AMD in the fellow eye is high, with almost half of eyes at risk requiring bilateral treatment by 3 years [7]. Due to the chronic nature of the disease, increasing prevalence and overwhelmed services, nAMD is associated with a significant treatment burden for patients, carers and medical retina services.

Macular changes related to ageing can broadly be classified into three groups: normal eyes, early AMD or late AMD (indeterminate, wet active, dry or wet inactive) $[8,9]$. Most visual loss happens in late nAMD because of progression of CNV [10]. In recent years, the importance of anatomical efficacy and its relation to disease control in patients with nAMD has become increasingly prominent. However, significant debate remains regarding how best to manage nAMD when assessing features of disease activity via optical coherence tomography (OCT), and particularly the significance of different types of fluid and how our understanding of anatomical efficacy (measurement of improvement regarding anatomical features guided by OCT assessment) can influence treatment strategies. Early randomised controlled trials measured both visual acuity and OCT changes to determine treatment efficacy and disease progression. We have since seen a 
resurgence in assessing disease control (a state where the choroidal neovascularisation is not causing impact on OCT parameters and visual function) and fluid in the macula, which have become a significant consideration in the management of patients with nAMD. However, a clear definition of disease control is required, as there is not a general consensus at present. The view point that some fluid is beneficial in patients with nAMD also needs to be addressed [11-14]. It is important to note whilst there is preliminary evidence that patients with untreated subretinal fluid (SRF) can still manage a good outcome despite this disease activity $[12,13]$, there is a misconception that leaving SRF untreated is in some way beneficial for the final outcome. The main aim in disease management should be to control all active disease and SRF can be a product of this activity so should be treated. Evans and colleagues analysed data from the CATT and IVAN studies and found a direct correlation between vision, fluid (of all types), the amount of fluid and fluctuations in CST, leading to the conclusion that variation in retinal thickness is adversely associated with visual outcome [14]. In addition, individual interpretation of how much fluid can be tolerated is largely based on the confidence and experience of the physician, and choosing to accept some fluid assumes the patient will be followed up and treated continuously, which may not be possible within services already under pressure.

The Retina Outcomes Group (ROG) is a forum group consisting of retinal specialists from a variety of NHS hospital ophthalmology departments, who aim to review contemporary issues in retinal disease therapy and provide expert consensus and guidance [3]. Meetings between the ROG (facilitated by Novartis UK) explored various aspects of disease control to provide insights into the importance of anatomical efficacy in nAMD. This led to the development of recommendations for healthcare professionals (HCPs) aimed at improving understanding and promoting best practice to achieve disease control. This article is not a review of the efficacy, safety, cost or convenience of specific therapies. Rather, the purpose of this article is to discuss the practical implications of anatomical efficacy and significance of fluid in the management of nAMD and propose recommendations for healthcare professionals to improve understanding and promote best practice to achieve disease control. An evidence-based review was performed, and an expert panel provided insights on a recommended UK definition for disease control and anatomical efficacy. The current evidence for the role and practical implications of anatomical efficacy and the significance of fluid at the macula in the management of patients with nAMD was also discussed. Visual acuity is a well-known outcome measure used in the assessment of nAMD, but will not be discussed in depth as anatomical efficacy is the focus of the current article.

This article is based on previously conducted studies and does not contain any new studies with human participants or animals, performed by any of the authors.

\section{Understanding the Impact of Fluid in the Retina of nAMD Patients}

The goal of treatment for patients with nAMD is to maximise visual gains, where feasible, and then maintain tight control of disease activity in the longer term. This helps to minimise erosion of vision due to nAMD activity. Studies have demonstrated that undertreatment of AMD leads to vision loss and demonstrate the importance of fluid as a key retreatment criteria (Table 1) [15]. In comparison, the absence of fluid on OCT and maintaining a dry retina correlate with vision gain $[16,17]$.

The subanalysis of the EXCITE trial aimed to identify the specific morphological effects of anti-angiogenic intervention across three different dosing regimens. Visual function was correlated to structural responses [28]. Alterations in intraretinal cysts (IRC), subretinal fluid (SRF) and pigment epithelial detachments (PEDs) significantly influenced the potential for visual gains [28].

The CATT study, a 2-year study of ranibizumab and bevacizumab in patients with nAMD $(N=1105)$, demonstrated that both drugs substantially and immediately reduced the amount 
Table 1 Retreatment criteria in nAMD clinical trials

\begin{tabular}{|c|c|c|}
\hline Trial & Regimen & Retreatment criteria \\
\hline $\begin{array}{r}\text { CATT } \\
{[18]}\end{array}$ & PRN & $\begin{array}{l}\text { Any fluid on OCT, new/ } \\
\text { persistent haemorrhage, } \\
\text { decreased VA vs. previous } \\
\text { exam, leakage and/or } \\
\text { increased lesion size on FA }\end{array}$ \\
\hline IVAN [19] & $\begin{array}{c}\text { Monthly } \\
\text { vs. } \\
\text { PRN }\end{array}$ & $\begin{array}{l}\text { Any SRF or increasing IRF on } \\
\text { OCT, new haemorrhage, VA } \\
\text { loss } \geq 10 \text { letters, fluorescein } \\
\text { leakage greater than only } 25 \% \\
\text { of lesion circumference or } \\
\text { expansion of CNV on FA }\end{array}$ \\
\hline $\begin{array}{c}\text { GEFAL } \\
{[20]}\end{array}$ & PRN & $\begin{array}{l}\text { Loss of } \geq 5 \text { letters with no } \\
\text { obvious atrophy or subretinal } \\
\text { fibrosis AND any fluid on } \\
\text { OCT, active "exudation" on } \\
\text { OCT (SRF unless stable since } \\
\text { the last } 3 \text { monthly injections } \\
\text { or macular edema with IRF, } \\
\text { or increase in CRT } \geq 50 \mu \mathrm{m} \\
\text { vs. previous exam), increased } \\
\text { CNV area/persistent leakage } \\
\text { on FA, new/persistent } \\
\text { subretinal or intraretinal } \\
\text { haemorrhage }\end{array}$ \\
\hline $\begin{array}{l}\text { HARBOR } \\
{[21]}\end{array}$ & PRN & $\begin{array}{l}\geq \text { 5-letter decrease from } \\
\text { previous visit AND any } \\
\text { evidence of disease activity } \\
\text { on OCT }\end{array}$ \\
\hline $\begin{array}{c}\text { LUCAS } \\
{[22]}\end{array}$ & $\mathrm{T} \& \mathrm{E}$ & $\begin{array}{l}\text { Any fluid on OCT, new/ } \\
\text { persistent haemorrhage, new/ } \\
\text { persistent dye leakage, or } \\
\text { increased lesion size on FA }\end{array}$ \\
\hline
\end{tabular}

Table 1 continued

\begin{tabular}{|c|c|c|}
\hline Trial & Regimen & Retreatment criteria \\
\hline VIEW [23] & PRN & $\begin{array}{l}\text { Any fluid on OCT, loss of } \geq 5 \\
\text { letters vs. best previous score } \\
\text { with recurrent fluid on OCT, } \\
\text { increase in CRT } \geq 100 \mu \mathrm{m} \\
\text { vs. lowest previous value, new- } \\
\text { onset classic } \\
\text { neovascularisation, new/ } \\
\text { persistent leakage on FA, new } \\
\text { haemorrhage }\end{array}$ \\
\hline $\begin{array}{l}\text { TREX- } \\
\text { AMD } \\
{[24]}\end{array}$ & $\mathrm{T} \& \mathrm{E}$ & $\begin{array}{l}\text { "Wet macula" (any unresolved } \\
\text { IRF/SRF on OCT), } \\
\text { subretinal/intraretinal } \\
\text { haemorrhage related to } \\
\text { exudative AMD }\end{array}$ \\
\hline $\begin{array}{l}\text { CAN- } \\
\text { TREAT } \\
{[25]}\end{array}$ & $\begin{array}{l}\text { Monthly } \\
\text { vs. } \\
\text { T\&E }\end{array}$ & $\begin{array}{l}\text { The presence of any fluid on } \\
\text { OCT, vision loss of }>5 \\
\text { ETDRS letters, presence of } \\
\text { new haemorrhage or } \\
\text { progression of choroidal } \\
\text { neovascularisation, or a } \\
\text { combination thereof }\end{array}$ \\
\hline $\begin{array}{c}\text { RIVAL } \\
\text { [26] }\end{array}$ & $\mathrm{T} \& \mathrm{E}$ & $\begin{array}{l}\text { Markers of disease activity: a loss } \\
\text { of VA of } \geq 5 \text { letters than the } \\
\text { best VA recorded since } \\
\text { treatment started (where VA } \\
\text { loss is considered, by the } \\
\text { investigator, to be due to } \\
\text { disease activity); new retinal } \\
\text { haemorrhage; the presence of } \\
\text { any IRF or SRF on OCT. } \\
\text { Dose adjustment: } 1 \text { criterion } \\
\text { present }=\text { interval reduced by } \\
2 \text { weeks; } \geq 2 \text { criteria } \\
\text { present }=\text { interval brought } \\
\text { back to } 4 \text { weekly }\end{array}$ \\
\hline
\end{tabular}


Table 1 continued

\begin{tabular}{lll}
\hline Trial & Regimen & Retreatment criteria \\
\hline ALTAIR & T\&E & New or persistent fluid with \\
[27] & unchanged or increased fluid \\
& volume on OCT, vision loss of \\
& $>5$ ETDRS letters, an \\
& increase in CRT \\
& of $\geq 100 \mu$ m at the central \\
& 1 mm compared with the \\
& lowest previous value \\
& measured by OCT, new-onset \\
& neovascularization based on \\
& fundus examination and \\
& multi-imaging assessment, \\
& new macular hemorrhage or \\
& new fluid or persistent intra- \\
& or subretinal fluid with \\
& unchanged or increased \\
& fluid volume from the \\
& previous visit as indicated by \\
& total OCT scan area
\end{tabular}

$C N V$ choroidal neovascularisation, $C R T$ central retinal thickness; ETDRS Early Treatment of Diabetic Retinopathy Scale, $F A$ fluorescein angiography, IRF intraretinal fluid, $O C T$ optical coherence tomography, $P R N$ pro re nata, $S R F$ subretinal fluid, $T \& E$ treat and extend, $V A$ visual acuity

of fluid in or under the retina [29]. The greater prevalence of fluid in the bevacizumab-as-needed group led to an average of 0.6 more injections during the 2 nd year than in ranibizumabas-needed patients and an average of 1.5 injections more over a 2-year period [30]. The associations between visual acuity and morphological features identified were maintained or strengthened in the 2nd year of treatment [31]. Under the pro re nata (PRN) regimen, tolerating fluid in patients with nAMD led to vision loss and poorer outcomes in year 2 . At week 104, eyes with intraretinal fluid (IRF) in the foveal centre had a lower visual acuity (VA) than eyes without intraretinal fluid (IRF, 59.9 vs. 70.9 letters; $P<0.0001)$. At week 104 , eyes with very thin $(<120 \mu \mathrm{m} ; 57.7$ letters $)$ or thick retinas ( $>212 \mu \mathrm{m} ; 64.0$ letters) had worse mean VA than eyes with normal retinal thickness (120-212 $\mu \mathrm{m} ; 72.0$ letters; $P<0.0001)$ [31].

A similar impact of fluid on vision loss was observed with the PRN regimen in the IVAN study $(n=525)$. The total lesion thickness and the neurosensory retinal thickness, including SRF, did not differ significantly between treatment groups, but were observed to be significantly lower for the continuous regimen patient groups compared with the discontinuous regimen groups [32]. Patients receiving continuous monthly treatment had slightly better visual function results compared with those receiving discontinuous treatment, although this improvement was not reflected in the primary outcome of best-corrected visual acuity (BCVA) or in self-reported health-related quality of life [32].

Recurrence of SRF has been also shown to be associated with VA loss in patients with nAMD. In a study from Wickremasinghe and colleagues, it was observed that not all vision loss is associated with new fluid and vision can decrease in the absence of any signs of fluid and that the recurrence of fluid often leads to vision loss [33]. New onset of fluid was more likely to lead to vision loss compared with patients who maintain a fluid-free macula or where there is stable but persistent fluid [33]. Overall, $29.6 \%$ of patients with recurring SRF lost five or more EDTRS letters between visits compared with $33.9 \%$ of patients with recurring IRF and $16.6 \%$ of patients with dry eyes $(P=0.001)$ [33]. In a separate study by Jaffe et al. [34], eyes with residual IRF were observed to have worse VA than those without.

Several other studies have assessed fluid levels and the impact these have on vision loss. Variations in thickness of CST have been associated with poorer vision outcomes [14, 35-37]. The HARBOR study highlighted that tolerating fluid can also lead to vision loss. A study by Chakravarthy et al. [11] demonstrated that the absence of IRF or SRF at $\geq 2$ visits post-loading dose is associated with better VA outcomes at 
Table 2 Summary of guidelines for nAMD and the use of OCT $[8,38-40]$

\begin{tabular}{ll}
\hline Guideline & Recommendation \\
\hline AAO 2014 & Early detection and prompt \\
& treatment of nAMD improves \\
& visual outcomes. Treatment \\
& with AREDS2 supplements \\
& reduces the progression to \\
& advanced AMD in the fellow \\
& eye \\
Fundus angiography and OCT & are useful diagnostic tests in \\
& clinical practice to detect new \\
& or recurrent neovascular \\
& disease activity and guide \\
& therapy \\
Offer OCT to people with & suspected late AMD (wet \\
active) & For eyes with confirmed late \\
AMD (wet active) offer \\
NICE NG82 2018
\end{tabular}

Table 2 continued

\begin{tabular}{|c|c|}
\hline Guideline & Recommendation \\
\hline EURETINA 2014 & $\begin{array}{l}\text { Patients who have received } \\
\text { treatment should be regularly } \\
\text { monitored, either monthly or } \\
\text { following an individualised } \\
\text { PRN or treat-and-extend } \\
\text { regimen. Follow-up visits } \\
\text { should include examination } \\
\text { for new onset of a decrease in } \\
\text { vision and/or persistent } \\
\text { metamorphopsia. BCVA tests } \\
\text { should be repeated using } \\
\text { identical procedures. Further } \\
\text { examination by OCT is } \\
\text { required if stereoscopic fundus } \\
\text { examination reveals clinical } \\
\text { signs of retinal oedema, } \\
\text { detachment of the retinal } \\
\text { pigment epithelia (RPE) or } \\
\text { haemorrhage } \\
\text { The effect of anti-VEGF therapy } \\
\text { can be monitored by non- } \\
\text { invasive SD-OCT alone }\end{array}$ \\
\hline
\end{tabular}

1 year compared to when IRF or SRF are absent at $\leq 1$ visits.

The ROG agreed that the evidence reviewed suggests better outcomes for patients if a dry macula is maintained. If patients lose disease control, then their vision may deteriorate to the extent that this vision loss cannot be recovered. As new therapies and technologies become available, drying the macula and maintaining disease control may become even more achievable. Drying the macula could allow treatment intervals to be extended and the treatment burden to be reduced.

\section{Guidelines}

The importance of fluid and the detection of fluid using imaging such as OCT is clearly 
highlighted in both national and international guidelines (Table 2) [8, 38-40].

There is currently a lack of clear consensus surrounding disease control and retreatment. The latest guidelines recommend that treatment decisions be based around the presence of fluid and to include OCT monitoring. BCVA alone is insufficient to detect recurrence of neovascular activity in nAMD [40].

For retreatment, clinicians may wish to refer to the retreatment criteria for fluid in the large clinical trials. OCT Fluid is a key retreatment criterion across the nAMD landmark clinical trials (Table 1) [19-26, 29]. In the CATT study, the protocol for treatment was evidence of disease activity (e.g., fluid on OCT), with no minimum threshold for retinal thickness; this allowed therapy to be more responsive to disease activity [29]. In the TREX-AMD study, patients were randomised to monthly injections or a treat and extend (T\&E) protocol. The T\&E protocol involved three initial injections and then the interval between treatments was based on exudative disease activity (treatment occurred no more frequently than every 4 weeks and no less frequently than every 12 weeks) [24].

\section{Assessing Fluid in the Clinic and Impact on Management Decisions}

The agreed definition for anatomical efficacy was the 'measurement of improvement regarding anatomical features guided by OCT assessment'. Anatomical efficacy is a clinical endpoint that can be measured in daily clinical practice by ophthalmologists for consideration in treatment decisions for patients with nAMD. If anatomical efficacy is measured regularly, it can provide a consistent endpoint over time to show if disease activity in the eye is improving, staying the same or declining. When reviewing treatment for a patient with nAMD, the presence or absence of fluid in the retina or subretinal zones should be considered, as well as the type of persistent fluid present. Anatomical efficacy testing is an objective measure, compared with functional efficacy (e.g. visual acuity), which can be a less consistent measure due to subjective factors (e.g. patient concentration/ level of focus, patient fatigue, changes in light conditions).

OCT is a quick, non-invasive and reproducible imaging tool used in nAMD to monitor structural changes including fluid and monitor response to anti-VEGF therapy [41-43]. However, interpretation of the results can be challenging, and various elements can impact the assessment of fluid seen on OCT. The PRONTO study was developed as a model for subsequent studies investigating OCT-guided treatment, for example, the SUSTAIN study $[44,45]$. The use of OCT in the diagnosis of AMD led to a re-definition of treatment paradigms and the importance of assessing fluid levels. In addition, OCT can be used to dynamically monitor changes in intraretinal, subretinal and sub-RPE fluid, supporting re-treatment decisions. There may be examples where fluid is tolerated and there is no vison loss but cases where this occurs should be in consultation with a senior treating clinician. This may be an important consideration in patients with persistent fluid but stable vision.

\section{Challenges of Assessing Fluid}

Interpretation of OCT and understanding of disease activity can be challenging and impacted by clinical and non-clinical factors. Clinical factors that impact the assessment of fluid and disease activity can include: poor image quality or segmentation; coexistant pathology, including epiretinal membrane, vitreomacular traction, atrophy, fibrosis, outer retinal tubulations, vitelliform lesions and other causes of fluid, including central serous retinopathy. There is also a poor correlation between vision and fluid and tendency to tolerate small amount of fluid in eyes with good vision $[11,14]$. OCT can be misinterpreted when there is presence of subretinal hyper-reflective material (SHRM), material that builds up between the retina and retinal pigment epithelia (RPE) [46]. Neovascular lesions with SHRM have been linked to poor VA outcomes caused by fibrotic scars and photoreceptor atrophy [46]. OCT can also be used to detect the presence of retinal pigment epithelial detachments (PEDs), associated with poor patient outcomes [46]. 
The ability of OCT to monitor and non-invasively assess the presence or absence of macula fluid, and the extent of macular neovascularisation, allows clinicians to better determine when to initiate anti-VEGF treatment and when to retreat $[46,47]$. Non-clinical considerations can include the variation in one clinician's review of OCT results compared with a second clinician or between reading centres. Variations between reading centres and treating physicians were reported in the CATT study in particular in eyes with intraretinal fluid that were untreated [48]. Further challenges could include individual clinician work capacity and pressure, the use or lack of clinic protocols, multiple team members of varied ability and patient attendance rates. In addition, patients can suffer from an incomplete response to antiVEGF treatment. This persistent disease activity is defined as persistent intraretinal, subretinal or subretinal pigment epithelium fluid, persistent or new haemorrhage and/or progressive lesion fibrosis, assesed after initial loading dose or after a period of sustained treatment [49].

\section{Practical Benefits of Anatomical Efficacy and a Fluid-Free Macula for Other Stakeholders Including Patients and Commissioners}

Patients benefit from clear communication about AMD and this may help relieve anxiety and improve patient adherence to treatment. Agents that dry the macula effectively improve outcomes and may lead to an improvement in the quality of life for patients due to better vision, fewer hospital visits and lower levels of treatment-related stress. Providing a broad pragmatic approach to patient care that can be implemented by services already under pressure is advised and can be considered an optimal approach to disease control as newer therapies become available.

Commissioners and service providers are interested in identifying and collecting reliable outcome measures to help understand value for money and quality of care and to objectively assess performance. The use of anatomical efficacy, for example, variations in thickness after loading treatment, anatomical stability of eyes or anatomical disease control, may provide a useful additional measure of the quality of services. The potential reduction in hospital visits could also have a positive effect on carers and the wider economy. This may lead to lower levels of work absenteeism, fewer hospital transport resources and a reduced carbon footprint if outcomes are maintained in the longterm.

\section{Recommendations from the ROG}

The consensus among the ROG members is that a zero-tolerance approach to the presence of fluid in patients with nAMD, aiming for a consistently fluid-free macula, should be adopted by medical retina services. The ROG has proposed recommendations for achieving disease control through a zero-tolerance approach to the presence of fluid in nAMD, as patients who avoid fluctuations in fluid at the macula have better visual outcomes. These recommendations are based on clinical evidence and the real-world experience of the ROG. This approach also facilitates more standardised protocol-based treatment strategies. The recommendations are as follows:

\section{Service Protocol}

- Develop a clear consensus-led protocol for the medical retina team

- Include protocols that allow early OCT review to check for response within a few weeks of injection (to confim if fluid represents active disease) OR establish if active fluid by cautious extension of treatment interval

- Flag on electronic patient record (EPR) the date when zero fluid was obtained for a particular patient as a reference point for future reviews, or if a dry macula was not obtained

- Consider including consultant level input for difficult cases (e.g. fluid mimickers) 


\section{Training}

- Promote consistent clinical training of allied HCPs, supported by oversight from retinal specialists with experience in assessing fluid on OCT scans

- Include education and training to distinguish between different types of fluid and mimickers and ensure appropriate assistance is sought from senior specialists if unsure

\section{Regimen}

- Consider reducing retreatment interval, proactive regimen and/or alternative drug treatment options in patients with persistent fluid in an attempt to achieve a fluid-free macula

- Consider a lower threshold to switching treatment and switching anti-VEGF therapies in patients with persistent fluid in an attempt to dry the macula. This threshold may need to be balanced against the associated increase in clinic visits to counsel and consent in high-throughput services

- Virtual clinics and proactive dosing regimens lend themselves to medical retina consultant-led quality assurance so that equivocal cases can be further graded by an experienced clinician

- Also reviewing at 2 weeks after an injection can "prove" whether the disease was indeed active (the "fluid" did resolve) PLUS benchmarking disease status in patients where two eyes are treated but not simultaneously-e.g. comment on EPR that the right eye was imaged at 2 weeks on a certain date and this is the gold standard disease control for that eye

\section{Multi-disciplinary Team (MDT)}

- Utilise an MDT forum to assess or review challenging cases with apparent persistent disease activity. Consider further multimodal imaging and MDT-based discussions at regular meetings to assess/examine the stability of fluid with experts to interpret the OCT

- Utilise a MDT forum to assess or review challenging cases with apparent persistent disease activity

\section{Engagement}

- Educate patient groups and commissioners on the importance of disease control and the potential impact on patient outcomes and service provision

\section{CONCLUSION}

It is the view of the ROG that focussing on anatomical outcomes and achieving zero tolerance of fluid in the macula results in better outcomes for treated patients with nAMD. Anatomical outcomes are considered paramount when assessing treatment outcomes in patients with nAMD. Drying the IRF/SRF is paramount as a marker of disease activity. If disease activity is reduced or slowed, this leads to better clinical outcomes and a more favourable prognosis for the patient. Targeting a dry macula in patients with nAMD and aiming for disease control are essential to improve outcomes. The recommendations illustrated by the ROG aim to promote best practice among HCPs and medical retina services to help improve patient outcomes.

\section{ACKNOWLEDGEMENTS}

The views and opinions of the authors are not necessarily those of Novartis or the publisher.

Funding. This article and the journal's Rapid Service Fee were sponsored by Novartis Pharmaceuticals UK Ltd. group which developed it were sponsored by Novartis Pharmaceuticals UK Ltd. All authors received honoraria, contributed to the development of the manuscript, and retained final control of the content and editorial decisions. 
Medical writing/Editorial assistance. Medical writing assistance was provided by Daniel Webber, Rock Unlimited. Novartis have checked that the content was factually accurate and balanced. Funding was provided by Novartis Pharmaceuticals UK Ltd. for honoraria and medical writing assistance.

Authorship. All named authors meet the International Committee of Medical Journal Editors (ICMJE) criteria for authorship for this article, take responsibility for the integrity of the work as a whole, and have given their approval for this version to be published.

Authors' Contributions. All named authors contributed to the initial concept and design of the manuscript, were involved in the roundtable discussions. In addition, all authors were involved in drafting and reviewing the manuscript during its development as well as final approval.

Disclosures. Louise Downey: Advisory board work for Novartis, Bayer, Allergan, Alimera, Bausch and Lomb, Roche; Speakers fees for Novartis, Bayer, Allergan, Alimera, Bausch and Lomb, Roche; Travel grants from Novartis Bayer Allergan; Bushra Mushtaq, Advisory board consultant and travel grants received from Novartis, Bayer, Alimera sciences; Athanasios Vardarinos, Speaker fees, Consultancy fees, hospitality and support with research and attending conferences from Novartis. Consultancy fees, hospitality and support attending conferences from BAYER; Yit Yang; Honoraria and speaker fees from: Novartis, Roche, Bayer, Allergan. Konstantinos Balaskas, Winfried Amoaku, Tomas Cudrnak: Advisory board work for Novartis, Bayer; Speaker fees for Novartis, Allergan, Alimera Sciences; Travel grants from Novartis, Bayer, Allergan, Alimera Sciences; Hemal Mehta, Markus Groppe, Sajjad Mahmood: Honoraria for consultancy, lecturing, travel, and clinical research from Novartis, Bayer; Clinical research support from Roche. Quresh Mohamed, Philip Severn received honoraria from Novartis Pharmaeuticals UK Ltd for their contribution to the roundtable meetings. The authors report no other conflicts of interest in this work.

Compliance and Ethics Guidelines. This article is based on previously conducted studies and does not contain any new studies with human participants or animals performed by any of the authors.

Open Access. This article is licensed under a Creative Commons Attribution-NonCommercial 4.0 International License, which permits any non-commercial use, sharing, adaptation, distribution and reproduction in any medium or format, as long as you give appropriate credit to the original author(s) and the source, provide a link to the Creative Commons licence, and indicate if changes were made. The images or other third party material in this article are included in the article's Creative Commons licence, unless indicated otherwise in a credit line to the material. If material is not included in the article's Creative Commons licence and your intended use is not permitted by statutory regulation or exceeds the permitted use, you will need to obtain permission directly from the copyright holder. To view a copy of this licence, visit http://creativecommons.org/licenses/by$\mathrm{nc} / 4.0 /$.

\section{REFERENCES}

1. NICE. Ranibizumab and pegaptanib for the treatment of age-related macular degeneration. 2008. www.nice.org.uk/guidance/ta155. Accessed 2020.

2. Owen CG, Jarrar Z, Wormald R, Cook DG, Fletcher $\mathrm{AE}$, Rudnicka AR. The estimated prevalence and incidence of late stage age related macular degeneration in the UK. Br J Ophthalmol. 2012;96(5): 752-6.

3. Amoaku W, Balaskas K, Cudrnak T, et al. Initiation and maintenance of a Treat-and-Extend regimen for ranibizumab therapy in wet age-related macular degeneration: recommendations from the UK Retinal Outcomes Group. Clin Ophthalmol. 2018;12: 1731-40.

4. Rahman F, Zekite A, Bunce C, Jayaram H, Flanagan D. Recent trends in vision impairment certifications 
in England and Wales. Eye (Lond). 2020;34(7): 1271-8.

5. Bressler NM, Treatment of Age-Related Macular Degeneration with Photodynamic Therapy Study G. Photodynamic therapy of subfoveal choroidal neovascularization in age-related macular degeneration with verteporfin: two-year results of 2 randomized clinical trials-tap report 2. Arch Ophthalmol. 2001;119(2):198-207.

6. Rosenfeld PJ, Brown DM, Heier JS, et al. Ranibizumab for neovascular age-related macular degeneration. N Engl J Med. 2006;355(14):1419-31.

7. Gale RP, Mahmood S, Devonport H, et al. Action on neovascular age-related macular degeneration (nAMD): recommendations for management and service provision in the UK hospital eye service. Eye (Lond). 2019;33(Suppl 1):1-21.

8. NICE. NG82. Age-related macular degeneration. 2018. www.nice.org.uk/guidance/ng82. Accessed 2020.

9. Ferris FL 3rd, Wilkinson CP, Bird A, et al. Clinical classification of age-related macular degeneration. Ophthalmology. 2013;120(4):844-51.

10. Cobos E, Recalde S, Anter J, et al. Association between CFH, CFB, ARMS2, SERPINF1, VEGFR1 and VEGF polymorphisms and anatomical and functional response to ranibizumab treatment in neovascular age-related macular degeneration. Acta Ophthalmol. 2018;96(2):e201-12.

11. Chakravarthy U, Pillai N, Syntosi A, Barclay L, Best C, Sagkriotis A. Association between visual acuity, lesion activity markers and retreatment decisions in neovascular age-related macular degeneration. Eye (Lond). 2020;2020:5.

12. Guymer RH, Markey CM, McAllister IL, et al. Tolerating subretinal fluid in neovascular age-related macular degeneration treated with ranibizumab using a treat-and-extend regimen: FLUID study 24-month results. Ophthalmology. 2019;126(5): 723-34.

13. Sadda SR, Tuomi LL, Ding B, Fung AE, Hopkins JJ. Macular atrophy in the HARBOR study for neovascular age-related macular degeneration. Ophthalmology. 2018;125(6):878-86.

14. Evans EN, Chakravarthy U, Reeves B. Associations between variation in retinal thickness and visual function. In: Paper presented at: ARVO2019; Vancouver; 2018.

15. Regillo CD, Brown DM, Abraham P, et al. Randomized, double-masked, sham-controlled trial of ranibizumab for neovascular age-related macular degeneration: PIER Study year 1. Am J Ophthalmol. 2008;145(2):239-48.

16. Brown D. Correlation between changes in OCT and visual acuity in the management of neovascular AMD. Retina Today. 2013;2013:73-5.

17. Brown DM, Tuomi L, Shapiro H, Pier Study G. Anatomical measures as predictors of visual outcomes in ranibizumab-treated eyes with neovascular age-related macular degeneration. Retina. 2013;33(1):23-34.

18. CATT Research G, Martin DF, Maguire MG, et al. Ranibizumab and bevacizumab for treatment of neovascular age-related macular degeneration: twoyear results. Ophthalmology. 2012;119(7):1388-98.

19. Investigators IS, Chakravarthy U, Harding SP, et al. Ranibizumab versus bevacizumab to treat neovascular age-related macular degeneration: one-year findings from the IVAN randomized trial. Ophthalmology. 2012;119(7):1399-411.

20. Kodjikian L, Souied EH, Mimoun G, et al. Ranibizumab versus bevacizumab for neovascular age-related macular degeneration: results from the GEFAL noninferiority randomized trial. Ophthalmology. 2013;120(11):2300-9.

21. Busbee BG, Ho AC, Brown DM, et al. Twelve-month efficacy and safety of $0.5 \mathrm{mg}$ or $2.0 \mathrm{mg}$ ranibizumab in patients with subfoveal neovascular age-related macular degeneration. Ophthalmology. 2013;120(5):1046-56.

22. Berg K, Pedersen TR, Sandvik L, Bragadottir R. Comparison of ranibizumab and bevacizumab for neovascular age-related macular degeneration according to LUCAS treat-and-extend protocol. Ophthalmology. 2015;122(1):146-52.

23. Richard G, Mones J, Wolf S, et al. Scheduled versus pro re nata dosing in the VIEW trials. Ophthalmology. 2015;122(12):2497-503.

24. Wykoff CC, Croft DE, Brown DM, et al. Prospective trial of treat-and-extend versus monthly dosing for neovascular age-related macular degeneration: TREX-AMD 1-year results. Ophthalmology. 2015;122(12):2514-22.

25. Kertes PJ, Galic IJ, Greve M, et al. Canadian treatand-extend analysis trial with ranibizumab in patients with neovascular age-related macular disease: one-year results of the randomized canadian treat-and-extend analysis trial with ranibizumab study. Ophthalmology. 2019;126(6):841-8.

26. Gillies MC, Hunyor AP, Arnold JJ, et al. Effect of ranibizumab and aflibercept on best-corrected visual acuity in treat-and-extend for neovascular 
age-related macular degeneration: a randomized clinical trial. JAMA Ophthalmol. 2019;137(4): 372-9.

27. Ohji M, Takahashi K, Okada AA, et al. Efficacy and safety of intravitreal aflibercept treat-and-extend regimens in exudative age-related macular degeneration: 52- and 96-Week Findings from ALTAIR: a randomized controlled trial. Adv Ther. 2020;37(3): 1173-87.

28. Simader C, Ritter M, Bolz M, et al. Morphologic parameters relevant for visual outcome during antiangiogenic therapy of neovascular age-related macular degeneration. Ophthalmology. 2014;121(6):1237-45.

29. Group CR, Martin DF, Maguire MG, et al. Ranibizumab and bevacizumab for neovascular age-related macular degeneration. $\mathrm{N}$ Engl $\mathrm{J}$ Med. 2011;364(20):1897-908.

30. Comparison of Age-related Macular Degeneration Treatments Trials Research G, Martin DF, Maguire MG, et al. Ranibizumab and bevacizumab for treatment of neovascular age-related macular degeneration: two-year results. Ophthalmology. 2012;119(7):1388-98.

31. Sharma S, Toth CA, Daniel E, et al. Macular morphology and visual acuity in the second year of the comparison of age-related macular degeneration treatments trials. Ophthalmology. 2016;123(4): 865-75.

32. Chakravarthy U, Harding SP, Rogers CA, et al. Alternative treatments to inhibit VEGF in age-related choroidal neovascularisation: 2-year findings of the IVAN randomised controlled trial. Lancet. 2013;382(9900):1258-67.

33. Wickremasinghe SS, Janakan V, Sandhu SS, AmirulIslam FM, Abedi F, Guymer RH. Implication of recurrent or retained fluid on optical coherence tomography for visual acuity during active treatment of neovascular age-related macular degeneration with a treat and extend protocol. Retina. 2016;36(7):1331-9.

34. Jaffe GJ, Martin DF, Toth CA, et al. Macular morphology and visual acuity in the comparison of agerelated macular degeneration treatments trials. Ophthalmology. 2013;120(9):1860-70.

35. Chong V. Ranibizumab for the treatment of wet AMD: a summary of real-world studies. Eye (Lond). 2016;30(2):270-86.

36. Holz FG, Tadayoni R, Beatty S, et al. Multi-country real-life experience of anti-vascular endothelial growth factor therapy for wet age-related macular degeneration. Br J Ophthalmol. 2015;99(2):220-6.
37. Vardarinos A, Gupta N, Janjua R, Iron A, Empeslidis $\mathrm{T}$, Tsaousis KT. 24-month clinical outcomes of a treat-and-extend regimen with ranibizumab for wet age-related macular degeneration in a real life setting. BMC Ophthalmol. 2017;17(1):58.

38. AAO. American Academy of Ophthalmology Retina/Vitreo Panel. Preferred practice pattern guidelines. age-related macular degeneration. 2015. www.aao.org/ppp. Accessed 2020.

39. RCOphth. Age-related macular degeneration: guidelines for management. 2013. http://www. rcophth.ac.uk/wp-content/uploads/2014/12/2013SCI-318-RCOphth-AMD-Guidelines-Sept-2013FINAL-2.pdf. Accessed 2020.

40. Schmidt-Erfurth U, Chong V, Loewenstein A, et al. Guidelines for the management of neovascular agerelated macular degeneration by the European Society of Retina Specialists (EURETINA). Br J Ophthalmol. 2014;98(9):1144-67.

41. Castillo MM, Mowatt G, Lois N, et al. Optical coherence tomography for the diagnosis of neovascular age-related macular degeneration: a systematic review. Eye (Lond). 2014;28(12):1399-406.

42. Castillo MM, Mowatt G, Elders A, et al. Optical coherence tomography for the monitoring of neovascular age-related macular degeneration: a systematic review. Ophthalmology. 2015;122(2): 399-406.

43. Wibbelsman TD, Pandit RR, Xu D, et al. Trends in retina specialist imaging utilization from 2012 to 2016 in the United States medicare fee-for-service population. Am J Ophthalmol. 2019;208:12-8.

44. Lalwani GA, Rosenfeld PJ, Fung AE, et al. A variabledosing regimen with intravitreal ranibizumab for neovascular age-related macular degeneration: year 2 of the PrONTO Study. Am J Ophthalmol. $2009 ; 148(1): 43-58$.

45. Cruz-Gonzalez F, Cabrillo-Estevez L, Lopez-Valverde G, Cieza-Borrella C, Hernandez-Galilea E, Gonzalez-Sarmiento R. Predictive value of VEGF A and VEGFR2 polymorphisms in the response to intravitreal ranibizumab treatment for wet AMD. Graefes Arch Clin Exp Ophthalmol. 2014;252(3): 469-75.

46. Rosenfeld PJ. Optical coherence tomography and the development of antiangiogenic therapies in neovascular age-related macular degeneration. Invest Ophthalmol Vis Sci. 2016;57(9):14-26.

47. Jindal A, Ctori I, Fidalgo B, Dabasia P, Balaskas K, Lawrenson JG. Impact of optical coherence tomography on diagnostic decision-making by UK 
community optometrists: a clinical vignette study. Ophthalmic Physiol Opt. 2019;39(3):205-15.

48. Toth CA, Decroos FC, Ying GS, et al. Identification of fluid on optical coherence tomography by treating ophthalmologists versus a reading center in the comparison of age-related macular degeneration treatments trials. Retina. 2015;35(7):1303-14.
49. Mettu PS, Allingham MJ, Cousins SW. Incomplete response to Anti-VEGF therapy in neovascular AMD: exploring disease mechanisms and therapeutic opportunities. Prog Retin Eye Res. 2020;2020:100906. 